\title{
Short and Long-Term Time Series Forecasting Stochastic Analysis for Slow Dynamic Processes
}

\author{
Julián Pucheta1, Carlos Salas², Martín Herrera², Cristian Rodriguez Rivero ${ }^{3}$, Gustavo Alasino ${ }^{4}$ \\ ${ }^{1}$ FCEFyN-Universidad Nacional de Córdoba, Córdoba, Argentina \\ ${ }^{2}$ FTyCA-Universidad Nacional de Catamarca, Catamarca, Argentina \\ ${ }^{3}$ Cristian University of California, Los Angeles, USA \\ ${ }^{4}$ Universidad Torcuato Di Tella, Buenos Aires, Argentina \\ Email: jpucheta@unc.edu.ar
}

How to cite this paper: Pucheta, J., Salas, C., Herrera, M., Rodriguez Rivero, C. and Alasino, G. (2019) Short and Long-Term Time Series Forecasting Stochastic Analysis for Slow Dynamic Processes. Applied Mathematics, 10, 704-717.

https://doi.org/10.4236/am.2019.108050

Received: July 25, 2019

Accepted: August 24, 2019

Published: August 27, 2019

Copyright ( 2019 by author(s) and Scientific Research Publishing Inc. This work is licensed under the Creative Commons Attribution International License (CC BY 4.0).

http://creativecommons.org/licenses/by/4.0/

\begin{abstract}
This paper intends to develop suitable methods to provide likely scenarios in order to support decision making for slow dynamic processes such as the underlying of agribusiness. A new method to analyze the short- and long-term time series forecast and to model the behavior of the underlying process using nonlinear artificial neural networks (ANN) is presented. The algorithm can effectively forecast the time-series data by stochastic analysis (Monte Carlo) of its future behavior using fractional Gaussian noise (fGn). The algorithm was used to forecast country risk time series for several countries, both for short term that is 30 days ahead and long term 350 days ahead scenarios.
\end{abstract}

\section{Keywords}

Stochastic Analysis, Time Series Forecasting, Decision Making, Dynamic Process, Process Modelling

\section{Introduction}

The agribusiness activities are the engine where the vegetal production lies with its decision-making built-in [1]. In Argentina, the activity's profit is subjected to a good production plan which in turn is subjected to financial variables [2]. One of them is the Emerging Market Bond Index (EMBI) known as country risk. This variable indexes the economic health of the country and is a strong signal when is compared against that of Chile, Brazil and Mexico countries whose products compete with those of Argentinian. When the producer must perform the production plan, there arises the need for counting with information about the EMBI values with some future horizon. In this paper, a method to forecast time series from EMBI with short and long term horizons is proposed. 
The article is structured as follows: after this Introduction, Section 2 describes an overview of the problem statement. Section 3 shows the proposed approach of the training and the selection method based on fractional Brownian motion using classical non-linear autoregressive model based on neural networks. Section 4 shows the sets of monthly EMBI series from Latin America countries, with emphasis on the statistical evaluation of the short-range case (800 training data samples) and the long-range (2700 training data). Section 5 presents some discussions and concluding remarks.

\section{Problem Formulation}

When agricultural venture is focused on profit from production purposes, several dynamic rates arises. One of them, the very short-term dynamic embraces process such as irrigations and nutrients, chemical applications in which it must take that decision [3]. Another dynamic arises when the producer plans the crop to sow and the rainfall forecast is required to perform such a task at an optimal date decision [4] [5] [6] [7]. However, the slow dynamic process related with the final product offer and demand is the variable with special interest in the agronomic venture which is subject to future economic scenario [8].

Is in this scenario where earn importance some economic variables, specifically the EMBI [9] whose role serves to measure the economic health of a country. So, in order to take optimal decision in the choice of crop and date, the availability of some economic future information about some player is required. Therefore, future scenarios from economic health in Argentina, Chile and Brazil in short and long term gives information to improve such decision making [10].

\section{Proposed Approach}

In this paper, a classical non-linear autoregressive model based on neural networks whose parameters are batch tuned [11] and its performance is evaluated by stochastic analysis is proposed [12].

After training is completed, predictions are generated by using Monte Carlo with Gaussian noise (Gn) and fGn [13]. The expectation from each prediction ensemble is computed for obtaining a deterministic time series both for Gn and $\mathrm{fGn}$. The stochastic analysis of these time series is performed for determining the forecast with the appropriate stochastic roughness characteristic and so it is chosen for the forecast. This section details the training and the selection method based on fGn.

\subsection{The Basic Problem}

The classical prediction problem can be formulated as follows. Given past values uniformly time-spaced of a process, namely $x(n-1), x(n-2), \cdots, x(n-N)$ where $N$ is the time series length, the present value $x(n)$ must be predicted.

Here, a prediction device is designed by considering the given sequence $\left\{x_{n}\right\}$ at time $n$ corresponding to a time series it can be obtained the best prediction $\left\{x_{e}\right\}$ 
for the following future 30/350 values sequence. Hence, it is proposed a predictor filter with an input vector $x$, which is obtained by applying the delay operator $\mathrm{D}$, to the sequence $\left\{x_{n}\right\}$. Then, the filter output will generate $x_{e}$ as the next value, that will be best estimate of the present value $x_{n}$. So, the prediction error at time $k$ can be evaluated as

$$
e(k)=x_{n}(k)-x_{e}(k)
$$

which is used for the learning rule to adjust the $\mathrm{NN}$ weights.

The predictor is implemented by an autoregressive neural network-based nonlinear adaptive filter. The NNs are used as a nonlinear model building to represent the underlying physical process dynamic behavior that generates the data. In this work, time lagged feed-forward networks are used.

The present value of the time series is used as the desired response for the adaptive filter, and the past values of the signal are supplied as adaptive filter input. Then, the adaptive filter output will be the one-step prediction signal. In Figure 1 the block diagram of the nonlinear prediction scheme based on a NN filter is shown. Therefore, our aim is to obtain the best prediction (in roughness sense) of the present values from a random (pseudo-random) time series.

\subsection{NN-Based AR Model}

Several experiences had been obtained from previous works detailed in [12]. Here, an NN-based AR filter model is tuned. The NN used is a time lagged feed-forward networks class. The NN topology consists of $l_{x}$ inputs, one hidden layer of $H_{o}$ neurons that are the processing nodes in the hidden layer, and one output neuron as shown [12]. The learning rule used in the learning process is based on the Levenberg-Marquardt method [14].

In order to predict the sequence $\left\{X_{e}\right\}$ one-step ahead, the first delay is taken from the tapped-line $x_{n}$ and used as input. Therefore, the output prediction can be denoted by

$$
x_{e}(n)=F_{p}\left(\left[\begin{array}{c}
x_{n-1} \\
x_{n-2} \\
\vdots \\
x_{n-1-l_{x}}
\end{array}\right], \boldsymbol{\xi}_{1}, \boldsymbol{\xi}_{2}\right)
$$

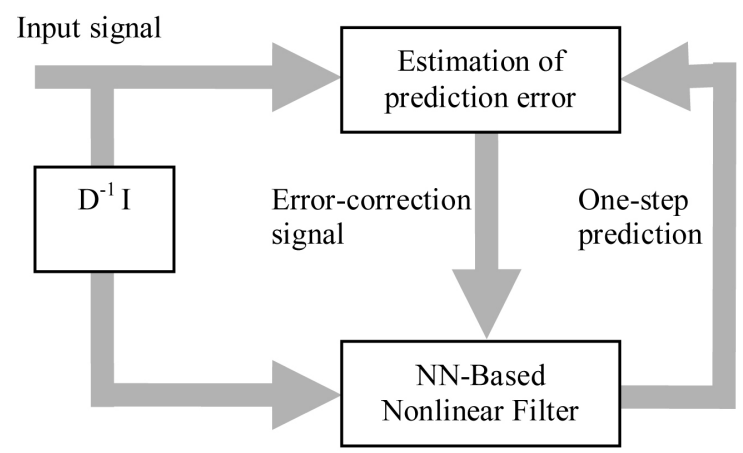

Figure 1. Block diagram of the neural network-based nonlinear predictor. 
where, $F_{p}\left(\cdot, \boldsymbol{\xi}_{1}, \boldsymbol{\xi}_{2}\right)$ is the nonlinear predictor filter with $I_{x}$ inputs and $x_{e}(n)$ is the output prediction at time $n$. In addition, $\xi_{1}$ and $\xi_{2}$ contain the tuning parameter composed by

$$
\xi_{1}=\left[\begin{array}{llll}
\xi_{1}^{1} & \xi_{1}^{2} & \cdots & \xi_{1}^{H_{o}}
\end{array}\right]
$$

whose elements are arrays defined as

$$
\xi_{1}^{i} \in \mathfrak{R}^{l_{x}+1}
$$

and

$$
\xi_{2} \in \mathfrak{R}^{H_{o}+1}
$$

where $H_{o}$ is the number of processing nodes. Thus, the predictor filter contains tuning parameters $\xi_{1}$ and $\xi_{2}$ that must be computed and from now on will not be explicit to avoid notation abuse.

\subsection{Monte Carlo Implementation Including Fractional Brownian Motion}

In this work the Hurst's parameter is used as statistical criteria for time series forecast by selecting the assessment in the algorithm. This $H$ gives an idea of signal roughness, and determines its stochastic dependence, here is implemented according to [15]. The definition of the Hurst's parameter appears in the Brownian motion from generalizing the integral to a fractional one. The Fractional Brownian motion $(\mathrm{fBm})$ is defined in the pioneering work by Mandelbrot [16] [17] through its stochastic representation

$$
B_{H}(t)=\frac{1}{\Gamma\left(H+\frac{1}{2}\right)}\left(\int_{-\infty}^{0}\left((t-s)^{H-\frac{1}{2}}-(-s)^{H-\frac{1}{2}}\right) \mathrm{d} B(s)+\int_{0}^{t}(t-s)^{H-\frac{1}{2}} \mathrm{~d} B(s)\right)
$$

where, $\Gamma(\cdot)$ represents the Gamma function

$$
\Gamma(\alpha)=\int_{0}^{\infty} x^{\alpha-1} \mathrm{e}^{-x} \mathrm{~d} x
$$

and $0<H<1$ is called the Hurst parameter. The integrator $B$ is a stochastic process, ordinary Brownian motion. The determination of $H$ results in a stochastic process with more roughness $(H<1 / 2)$ or more smoothness $(H>1 / 2)$ which is fixed given the real processes evidences. Note, that $B$ is recovered by taking $H=1 / 2$ in (6). Here, it is assumed that $B$ is defined on some probability space $(\Omega, F, P)$, where $\Omega, F$ and $P$ are the sample space, the sigma algebra (event space) and the probability measure respectively. Thus, an $\mathrm{fBm}$ is a time continuous Gaussian process depending on the so-called Hurst parameter $0<H<1$. The ordinary Brownian motion is generalized to $H=0.5$, and whose derivative is the white noise. The $\mathrm{fBm}$ is self-similar in distribution and the variance of the increments is defined by

$$
\operatorname{Var}\left(B_{H}(t)-B_{H}(s)\right)=v \cdot|t-s|^{2 H}
$$

where $v$ is 1 and $s=0$. 
This special form of the variance of the increments suggests various ways to estimate the parameter $H$. In fact, there are different methods for computing the parameter $H$ associated to Brownian motion [17]. In this work, the algorithm uses a wavelet-based method for estimating $H$ from a trace path of the fBm with parameter $H$ [18]. To generate data, as example, three ensemble from fBm with different values of $H$ are shown in Figure 2, where can be noted the difference in the variances for each $H$. The figure shows synthetized traces using the method described in [13], where the black dots corresponds to five traces for each $H$, the magenta lines shows the theoretical variances using (8) and the blue lines shows the variance estimated by

$$
\operatorname{Var}\left(f_{t}\right)=\frac{1}{N} \sum_{\omega}\left(f_{t, \omega}-E\left[f_{t}\right]\right)^{2}
$$

where $N$ is the size of $f(t, \omega)$ for each time $t$. and $\omega$ indicates each trace.

Finally, in this paper the method implemented in [15] was used.

\subsection{Algorithm Description}

Our thesis asserts that if some process evolves along time with any $H$, it will do in the future with the same $H$ namely keeping its smoothness or roughness. To do so, a classic non-linear model based on neural networks batch-tuned is proposed. In the tuning process, data are randomly split for generalizing and training, with a $15 \%$ and $85 \%$ ratio, respectively. Furthermore, since the last data are the most important given the series nature, the last or the last two are left for
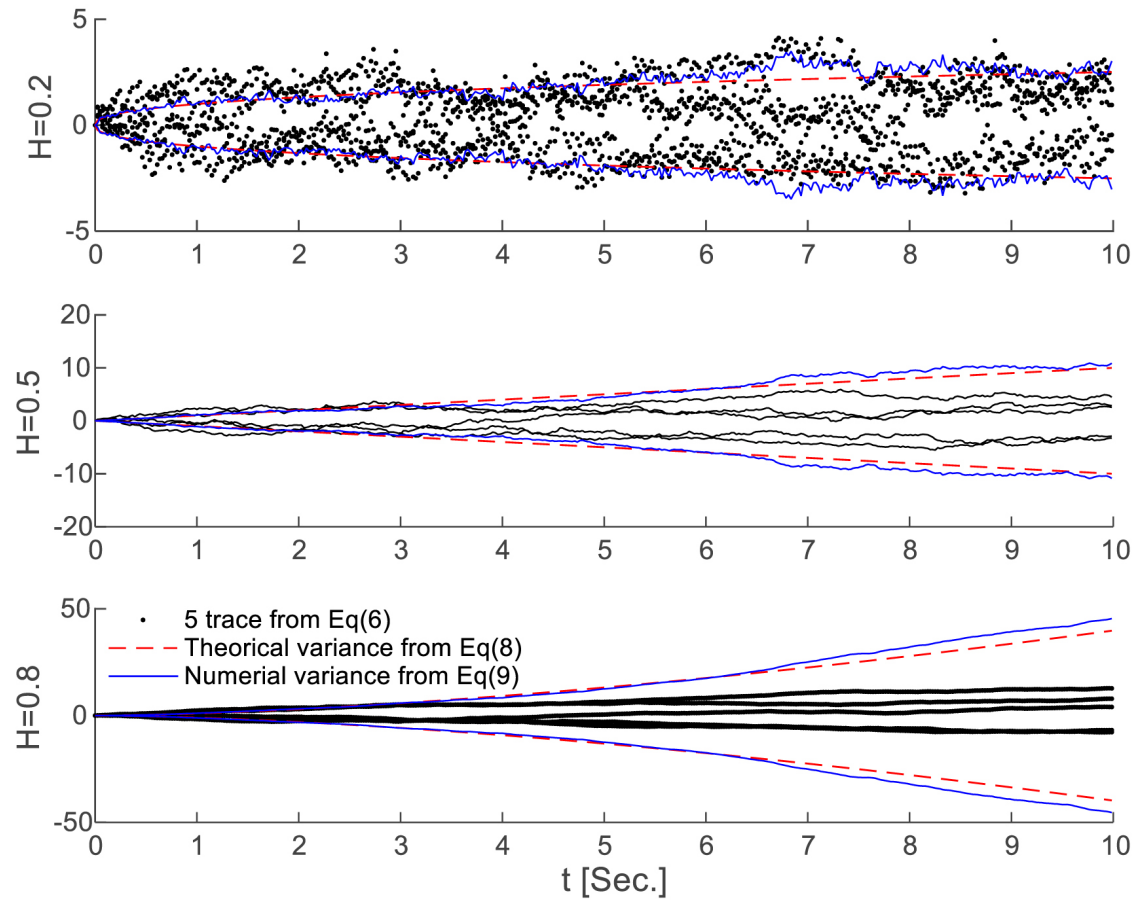

Figure 2. Five sample paths from fractional Brownian motion for three values of $H$ (computed over 50-trace ensemble), theoretical variances explicit in Equation (8) and numerical variance computed by Equation (9) are also depicted. 
evidence test. Thus, the relevant data quantity to be considered from the series is them determined for performing the desired forecast. By this way the parameters for Equation (3) are obtained.

After tune the filter defined in Equation (2), the predictions are generated using normal Gaussian and fractional Gaussian noise. In order to generate the ensemble with $R$ traces for forecasting the time series, it is modified the Equation (2) by

$$
x_{e}(n+q, \omega)=F_{p}\left(\left[\begin{array}{c}
x_{n-1+q} \\
x_{n-2+q} \\
\vdots \\
x_{n-1+q-l_{x}}
\end{array}\right], \omega\right)+\theta \cdot \Delta B_{H}(q, \omega)
$$

where $q=1,2, \cdots, F_{H}$ sets the future time, $F_{H}$ the forecast horizon, $\omega=1,2, \cdots, R$ denotes each trace, $R$ is the ensemble size and $\theta$ is a parameter for denormalizing $B_{H}$. For selecting a fixed value for $\theta$, one must take into account the length of the forecast horizon $F_{H}$, which in this work are 30 and 350 days and also the series dynamic range, using $\quad \theta=\left(\frac{1}{F_{H}} \sqrt{\left(\frac{\max (x)-\min (x)}{\max (x)}\right)}\right)^{2 H}$

and specifically when $H=0.5$ the Gaussian case is obtained. The coefficient $\theta$ was introduced for normalizing the ensemble along the forecast time. The ensemble described by Equation (10) is analyzed by classical statistical methods for obtaining the mean and the variance functions.

Once the tuning process is completed, for the short term forecast case a sequences pair is defined. One sequence is

$$
\left\{x_{n}\right\}, n=1,2,3, \cdots, N
$$

and the other is composed by the former concatenated with the forecasted which is the ensemble expectation from Equation (10), that is

$$
\left\{\left\{x_{n}\right\},\left\{E\left[x_{e}\right]\right\}\right\}, e=1,2,3, \cdots, F_{H}
$$

Both sequences are analyzed by the method detailed in [18] and such estimated $H$ must match between (12) and (13). Thus, ensemble mean of each prediction is taken and that one with the suitable stochastic roughness $H$ is chosen as the forecast. Table 1 details the method for tuning and selecting based on $\mathrm{fBm}$.

\section{Implementation with EMBI Time Series}

The method has been implemented by considering that there are more than 2700 data values from several countries.

Here it is proposed to build a model for forecasting the time evolution of the next 30 data following the current time-which is going to be considered as short range, and the next 350 values that corresponds to the long range. For both cases 
Table 1. Algorithm that performs the short/long term time series forecast.

1. Set the neural network (NN) topology by assigning $l_{x}, H_{o}$ Equation (4) Equation (5).

2 .

Chose the data for truth test that are just one or two of the last time series data that will not be used by training or validating processes.

3. Train the NN with $85 \%$ of the data and check the overfitting with the remaining $15 \%$.

4. Stop the training if the generalization error increases.

5. If the truth test data is not suitably forecasted, go to 1 .

6. Set $H=0.5$, set $\theta$ via Equation (11).

7. Run Monte Carlo including $H$ described by Equation (10).

8. Select the mean value that gives the best match of $\mathrm{H}$ according to the long or the short range scenario.

9. The obtained mean series generated and its variance are the algorithm results.

10. Given the case that the results were not credible, go to 6 and modify the noise characteristic by changing $H, 0<H<1$.

the methodology uses a batch training with a validation set of $15 \%$ of the random data set. In the short-range case, 800 training values were used and for the long-range, 2700.

The last group of one to three data was left as a test of disruptive validation or truth test, mainly for studying the overfitting. The prediction was made by Monte Carlo simulation where the noise used were stationary, seasonal and pseudorandom, although with a roughness characteristic determined by the Hurst parameter, generated by using [15].

The roughness is evaluated by the Hurst's parameter $H$, which is computed using a wavelet-based method [18]. After the tuning process is completed, two sequences pairs are defined. One pair is

$$
\left\{x_{n}\right\}, n=1,2,3, \cdots, N_{S}
$$

with

$$
\left\{\left\{x_{n}\right\},\left\{x_{e}\right\}\right\}, e=1,2,3, \cdots, F_{S}
$$

for the short term forecast given $N_{S}=800, F_{S}=30$. The other pair is

$$
\left\{x_{n}\right\}, n=2350,2351, \cdots, N_{L}
$$

with

$$
\left\{\left\{x_{n}\right\},\left\{x_{e}\right\}\right\}, e=1,2,3, \cdots, F_{L}
$$

for the long term forecast given $N_{L}=2700, F_{S}=350$. The selection of $N_{L}$ was determined given the available data samples, and the parameter $F_{S}$ was determined given that one year ahead is long term for development countries. Each pair of sequences must show the same $H$ parameter estimated by [18].

\subsection{Monthly EMBI Forecast}

The algorithm detailed in Table 1 was used to forecast short term time series of 
30 step ahead. The time series belong to EMBI from Argentina, Chile, Brazil and Mexico. The results are summarized in Table 2, and the evolution over time is shown in Figures 3-6. The images shown a shaded area that indicates the ensemble range, $100 \%$ of the traces, obtained by Monte Carlo. Inside the shaded area is

Table 2. Roughness results with the implementation of the 30-day algorithm (March 2019).

\begin{tabular}{ccccc}
\hline Series & Argentina & Chile & Brazil & Mexico \\
\hline$\left\{x_{n}\right\}$ & 0.66917 & 0.503 & 0.63827 & 0.65115 \\
$\left\{X_{m}, X_{e}\right\}$ fGn & 0.66941 & 0.49902 & 0.63864 & 0.65077 \\
& Figure 3(a) & Figure 4(a) & Figure 5(a) & Figure 6(a) \\
$\left\{x_{n}, X_{e}\right\}$ Gn & $\mathbf{0 . 6 6 8 9 5}$ & $\mathbf{0 . 5 0 2 2 7}$ & 0.6376 & $\mathbf{0 . 6 5 1 2 8}$ \\
& Figure 3(b) & Figure 4(b) & Figure 5(b) & Figure 6(b) \\
\hline
\end{tabular}

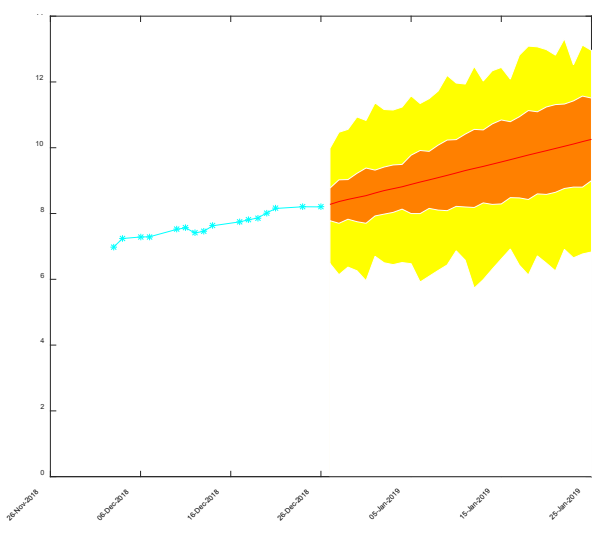

(a)

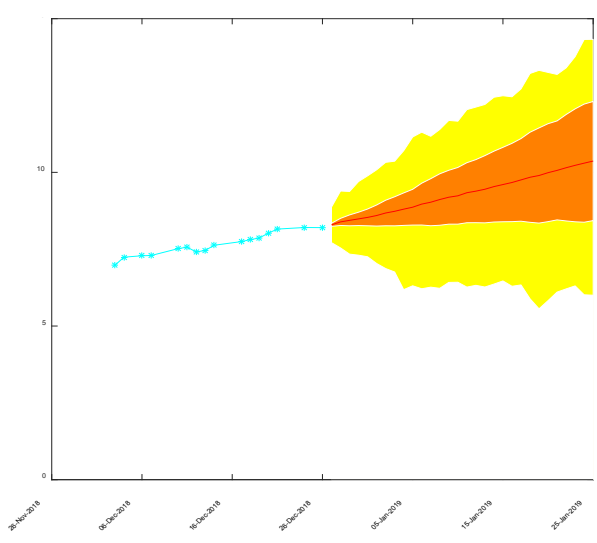

(b)

Figure 3. Argentinean EMBI forecast and its stochastic roughness up to Jan 2019. The cyan line is the data samples, the yellow area depicts the $100 \%$ of the Monte Carlo traces, the orange area indicates the $66 \%$ of the traces, and the red line is the mean value of the stochastic process. (a) $H=0.66941$; (b) $H=0.66895$.

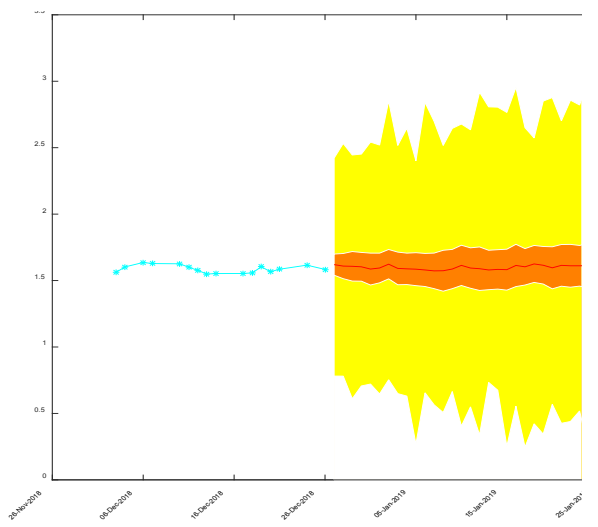

(a)

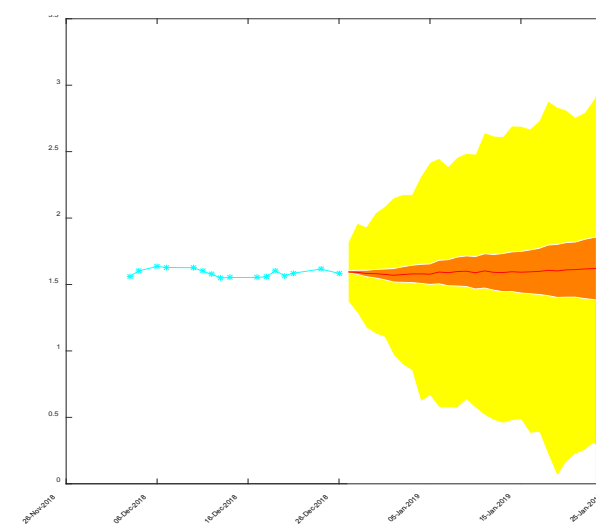

(b)

Figure 4. Chilean EMBI forecast and its stochastic roughness up to Jan 2019. (a) $H=0.49902$; (b) $H=0.50227$. 


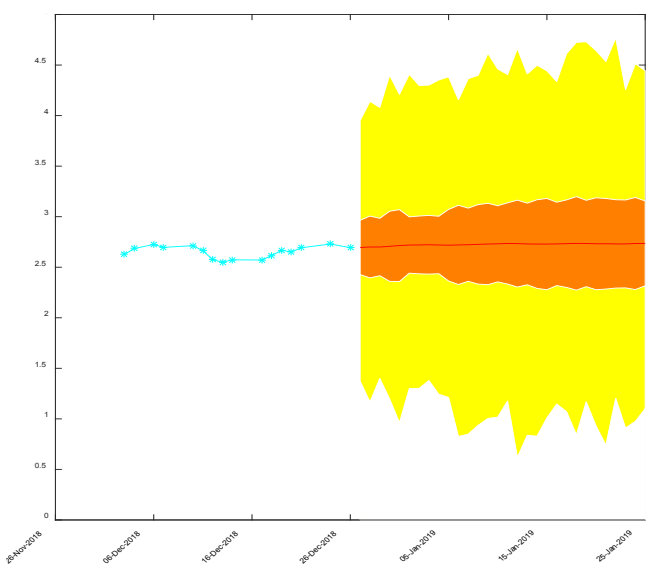

(a)

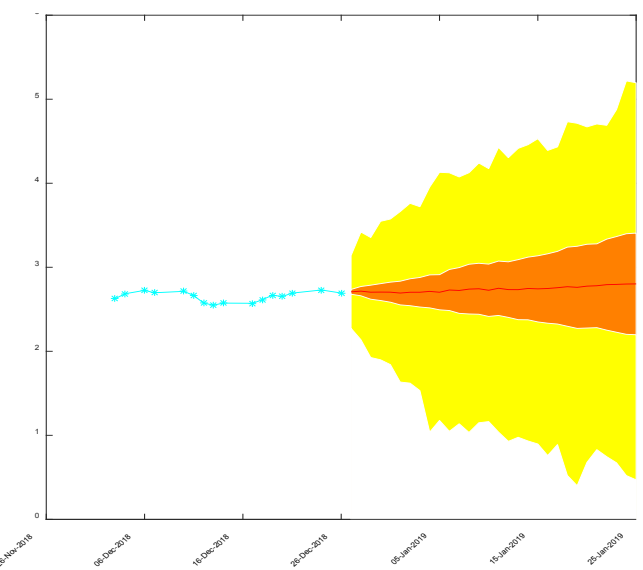

(b)

Figure 5. Brazilian EMBI forecast and its stochastic roughness up to Jan 2019. (a) $H=0.63864$; (b) $H=$ 0.6376 .

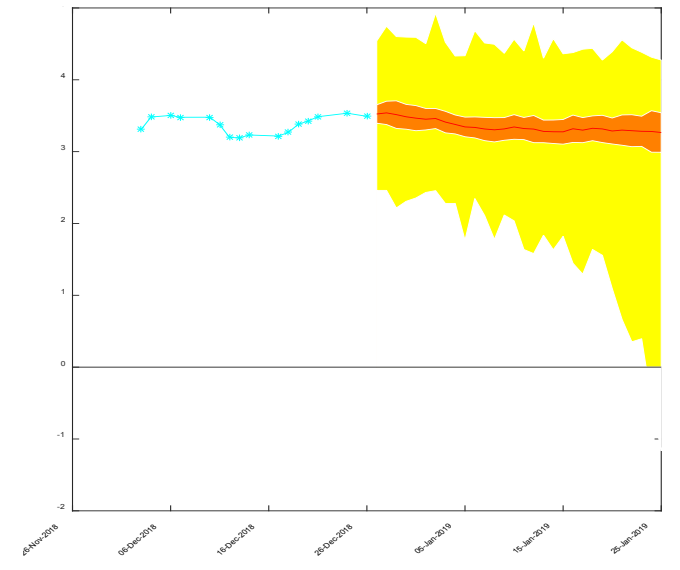

(a)

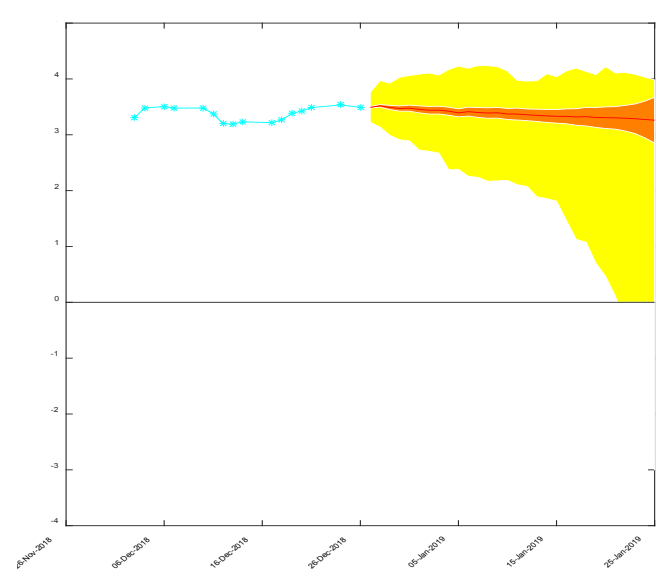

(b)

Figure 6. Mexican EMBI forecast and its stochastic roughness up to Jan 2019. (a) $H=0.65077$; (b) $H=$ 0.65128 .

another shaded area that indicates the $66 \%$ of the traces. The statistic indicator is $H$, which is detailed under each ensemble. The aim of show values of $H$, cases (a) and (b) of each series, is to highlight the behavior of the forecast. The case of the ensemble generation with $H=0.5$ is shown in the lower rows of the table, given that the estimation of $H$ gives figures near 0.5. In Table 2 are summarized the results, where the values in bold fonts indicates the forecast recommended by the criteria stated in this work.

\subsection{Annual EMBI Forecast}

The algorithm of Table 1 is used for forecasting long term periods, in this case 350 steps ahead. The simulation conditions are those of Equation (16) and Equation (17). Here, the Monte Carlo was set with fractional Gaussian noise and normal Gaussian noise, detailed in different rows of Table 3. The figures in bold 
Table 3. Roughness results with the implementation of the algorithm for 350 days hori-

zon

(a)

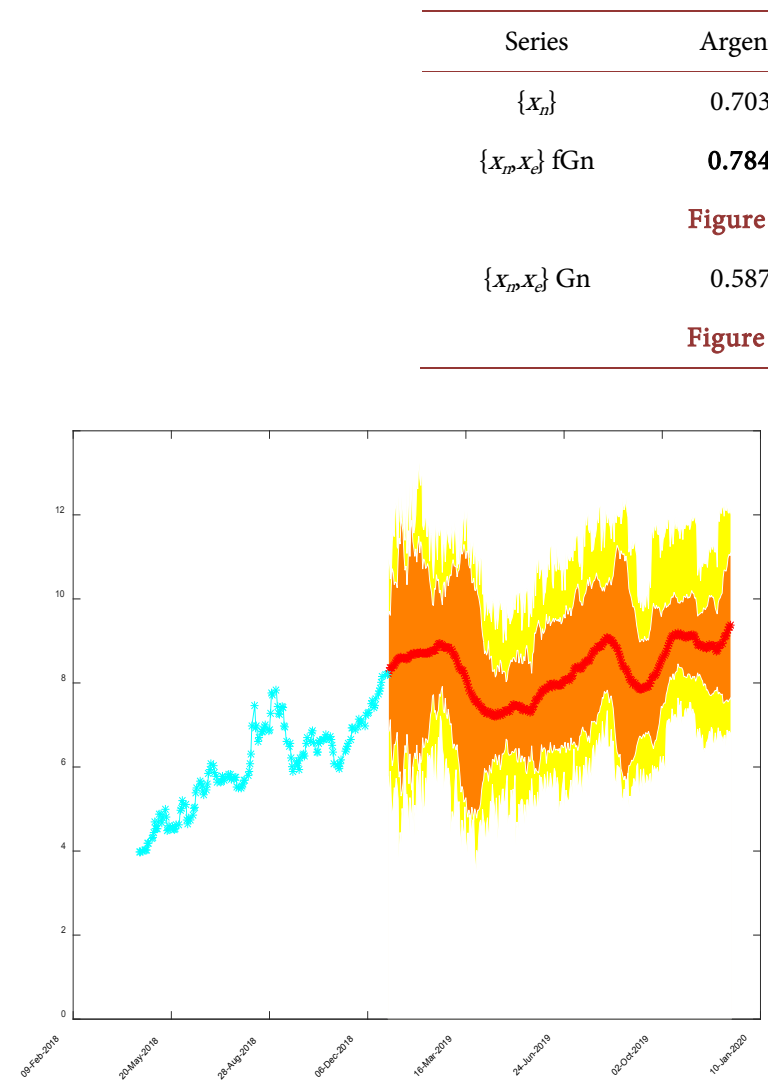

forecast

(Dec

2019).

\begin{tabular}{ccccc}
\hline Series & Argentina & Chile & Brazil & Mexico \\
\hline$\left\{X_{n}\right\}$ & 0.70392 & 0.40106 & 0.647 & 0.54395 \\
$\left\{X_{n}, X_{e}\right\}$ fGn & $\mathbf{0 . 7 8 4 2 5}$ & 0.6938 & $\mathbf{0 . 6 4 4 4}$ & $\mathbf{0 . 2 9 2 3 3}$ \\
& Figure 7(a) & Figure 8(a) & Figure 9(a) & Figure 10(a) \\
\multirow{2}{*}{$x_{n}, x_{e}$} Gn & 0.58782 & $\mathbf{0 . 4 7 2 1 3}$ & $\mathbf{0 . 4 4 1 7 2}$ & 0.83236 \\
& Figure 7(b) & Figure 8(b) & Figure 9(b) & Figure 10(b) \\
\hline
\end{tabular}

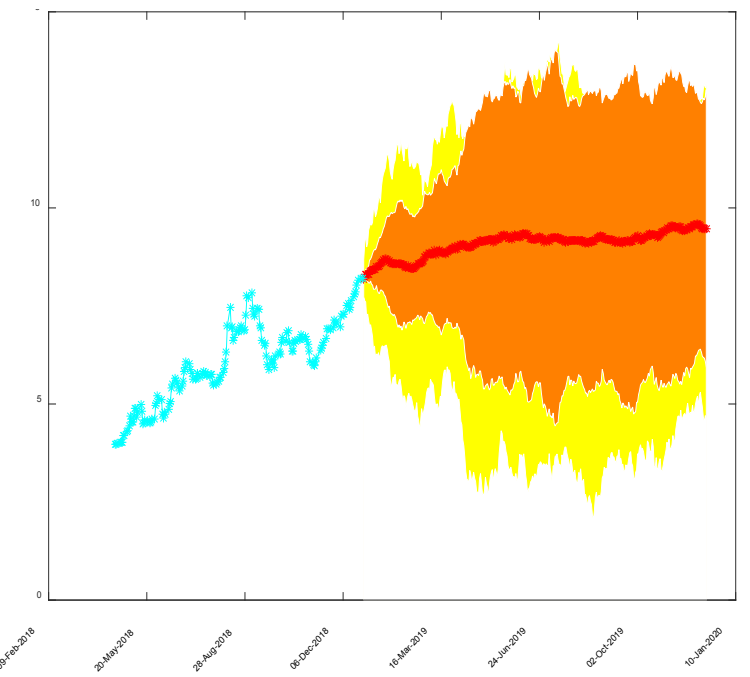

(b)

Figure 7. Argentinean EMBI forecast and its stochastic roughness up to Dec 2019. The cyan line is the data samples, the yellow area depicts the $100 \%$ of the Monte Carlo traces, the orange area indicates the $66 \%$ of the traces, and the red line is the mean value of the stochastic process. (a) $H=0.78425$; (b) $H=0.58782$.

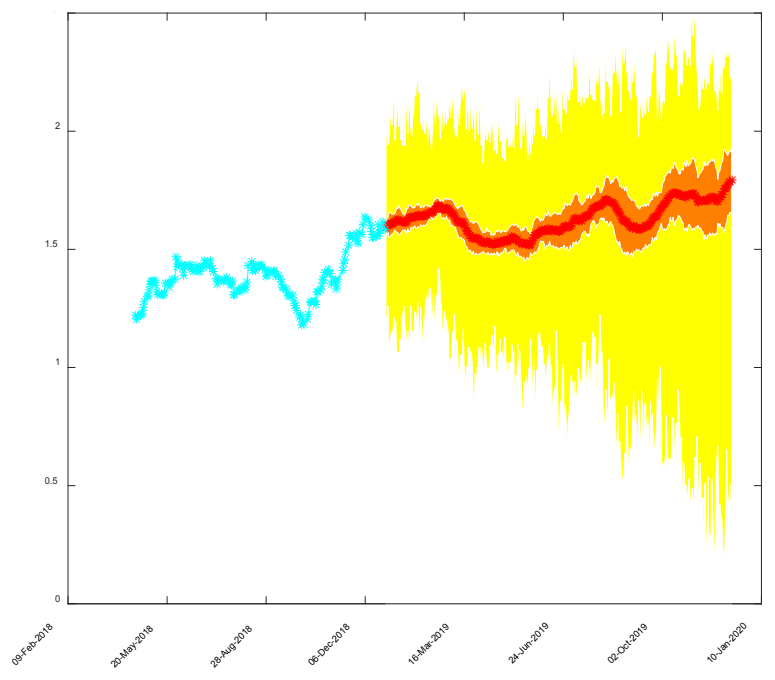

(a)

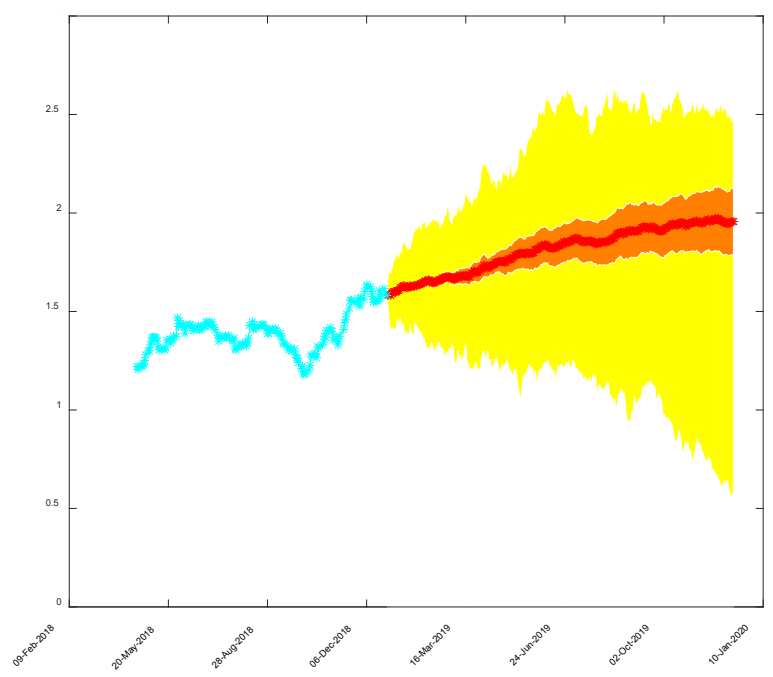

(b)

Figure 8. Chilean EMBI forecast and its stochastic roughness up to Dec 2019. (a) $H=0.6938$; (b) $H=0.47213$. 


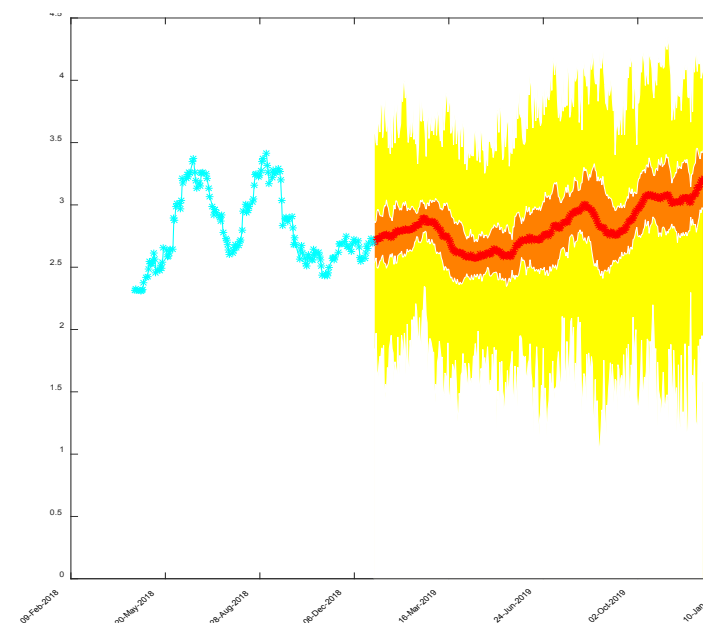

(a)

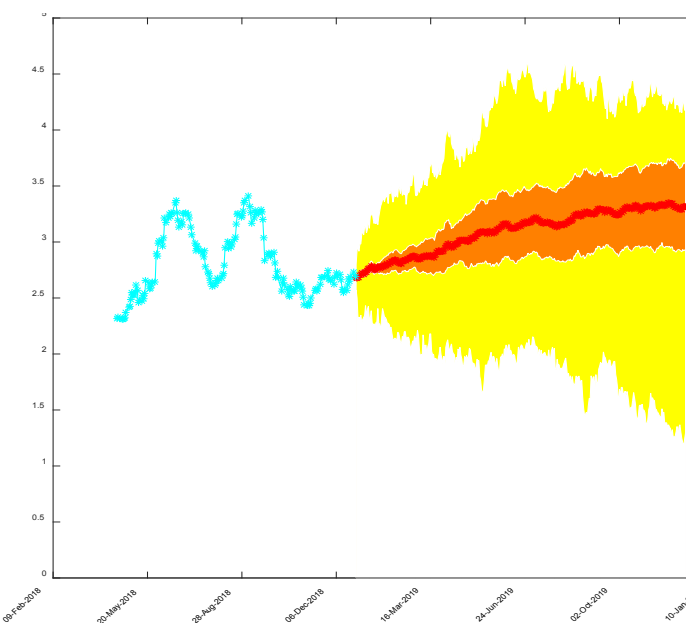

(b)

Figure 9. Brazilian EMBI forecast and its stochastic roughness up to Dec 2019. (a) $H=0.6444$; (b) $H=0.44172$.

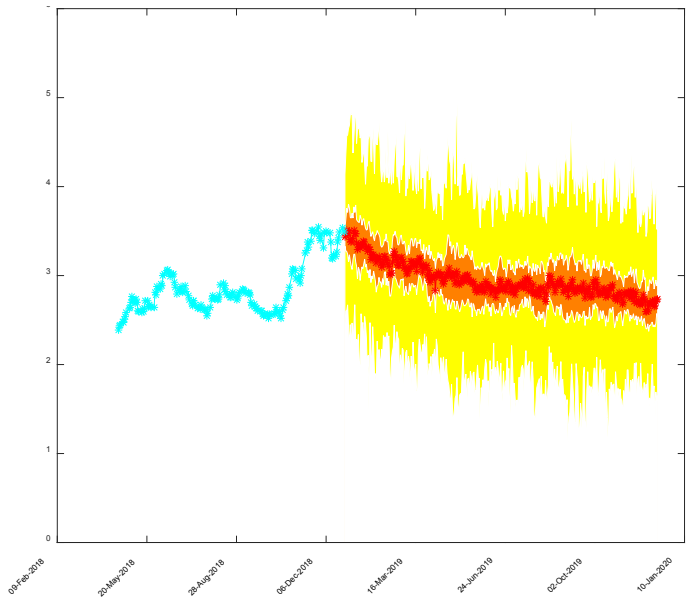

(a)

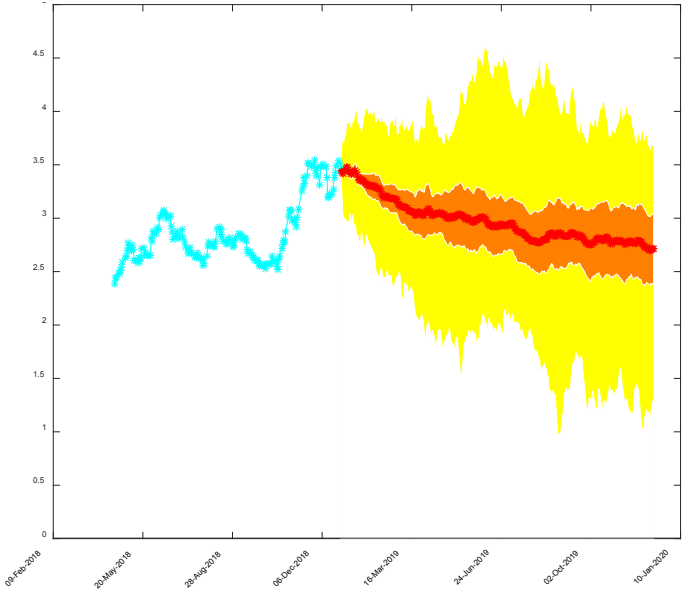

(b)

Figure 10. Mexican EMBI forecast and its stochastic roughness. (a) $H=0.29233$; (b) $H=0.83236$.

show the preferred forecast of this method. The shaded areas embrace the traces from the ensemble, where the darkest indicates the $66 \%$ of confidence and the continuous thick line is the mean value.

\subsection{Discussion}

From our results, the obtained EMBI behavior is stable with increasing trend in the Argentina's case, which is very volatile. The EMBI behavior shows a decreasing trend in the Mexico's case. The variation ranges estimated for Argentina is very broad although by tuning the $H$ parameter it can be narrowed. At the regional level, the variations of Chile and Brazil forecasted EMBI are moderate and the one of Mexico are very small.

The trend indicates a fall in the quarter and then continues declining respect of the range the historical. Volatility affects the amplitude of the range with pre- 
dicted values with probable values of descent around 495 basis points (bp) in less than 90 days.

The scenario of variations is high, but with a decreasing trend with wide margins conditioned by the high fluctuation of its historical index behavior. The average monthly value is around $700 \mathrm{bp}$ and with an annual downward trend. This is an acceptable forecast given that 2019 is a year with scheduled presidential elections in Argentina.

For Chile's case, the study starts around $165 \mathrm{bp}$ with an upward, soft and constant trend that continues up to the end of March with 160 points, which indicates some internal or external factor that slightly presses the upside. For its part, according to this model Brazil will start the year around $272 \mathrm{bp}$ and it would have an ascending behavior no higher than 290 until March 20 when it starts to return to 260, with small variation and regular movements without brusque highs and lows. Finally, in the case of Mexico, the prediction that it would begin in the range $350-360 \mathrm{bp}$, with a smooth descending trend towards the end of January 330 - $300 \mathrm{bp}$, and the rest of the quarter remains in 325 - 350 is also acceptable.

It can be concluded that the likely economic scenarios for agribusiness producers is competitive but very similar to that of prior years, so taking care this highlights the final product will be well positioned.

\section{Conclusions}

In this work, a methodology based on neural networks filter to model the underlying process behavior that causes the EMBI measurements evolution of the short and long term was detailed. The methodology consists of generating an artificial intelligence based predictor filter with a stochastic analysis of its future behavior using fractional Gaussian noise. Results of this analysis were shown to determine which series is the most coincident according to the Hurst parameter.

The generated information is not the exact value, but gives an idea about which will be the index trend based on its historical values intended for slow dynamic processes. The incorporation of this variable into decision making by estimating the predicted values impacts on the business plan or the investment portfolio. In the latter case, the EMBI provides a daily measure of the investor's perception regarding country risk. Thus, given the alarm sense that brings the EMBI rise, it is relevant to generate the projection of this series for the region and some countries that comprise it. Based on data predictions, it is possible to optimize the exposure in instruments, both bonds as shares, at country level and help to reduce the exposure in moments that anticipate an EMBI increase.

By this way, the proposed predictor model identifies relationships between different factors that allow assessing associated risks or likelihood based on a set of conditions. It guides the decision maker during the operations of the organization, identifying the probability of occurrence of events and the consequent value of the index in the short and medium term. It generates clarity on the sce- 
nario of the required return rate in dollars of the countries under analysis and allows the portfolio manager to reaffirm investment and exposure objectives considering the individuality of the risk of each country and the whole, or reformulate the investment.

\section{Conflicts of Interest}

The authors declare no conflicts of interest regarding the publication of this paper.

\section{References}

[1] Car, N.J. (2018) USING Decision Models to Enable Better Irrigation Decision Support Systems. Computers and Electronics in Agriculture, 152, 290-301. https://doi.org/10.1016/j.compag.2018.07.024

[2] Rupnik, R., Kukar, M., Vračar, P., Košir, D., Pevec, D. and Bosnić, Z. (2018) AgroDSS: A Decision Support System for Agriculture and Farming. Computers and Electronics in Agriculture, 161, 260-271. https://doi.org/10.1016/j.compag.2018.04.001

[3] Prabakaran, G., Vaithiyanathan, D. and Ganesan, M. (2018) Fuzzy Decision Support System for Improving the Crop Productivity and Efficient Use of Fertilizers. Computers and Electronics in Agriculture, 150, 88-97. https://doi.org/10.1016/j.compag.2018.03.030

[4] Alugubelly, M., Polepalli, K.R., Gade, S. and Ninomiya, S. (2019) Analysis of Similar Weather Conditions to Improve Reuse in Weather-Based Decision Support Systems. Computers and Electronics in Agriculture, 157, 154-165. https://doi.org/10.1016/j.compag.2018.12.010

[5] Li, Z.H., He, J.Q., Xu, X.G., Jin, X.L., Huang, W.J., Clark, B., Yang, G.J. and Li, Z.H. (2018) Estimating Genetic Parameters of DSSAT-CERES Model with the GLUE Method for Winter Wheat (Triticum aestivum L.) Production. Computers and Electronics in Agriculture, 154, 213-221. https://doi.org/10.1016/j.compag.2018.09.009

[6] Rivero, C.R., Pucheta, J., Sauchelli, V. and Patiño, H.D. (2016) Short Time Series Prediction: Bayesian Enhanced Modified Approach with Application to Cumulative Rainfall Series. International Journal of Innovative Computing and Applications, 7, 153-162. https://doi.org/10.1504/IJICA.2016.078730

[7] Rivero, C.R., Patiño, D., Pucheta, J. and Sauchelli, V. (2016) A New Approach for Time Series Forecasting: Bayesian Enhanced by Fractional Brownian Motion with Application to Rainfall Series. International Journal of Advanced Computer Science and Applications, 7, 237-244. https://doi.org/10.14569/IJACSA.2016.070334 http://thesai.org/Publications/ViewPaper?Volume $=7 \&$ Issue $=3 \&$ Code $=$ ijacsa $\&$ Serial $\mathrm{No}=34 \#$ sthash.ETZInPQR.dpuf

[8] Cavanagh, J. and Long, R. (1999) Introducing the J.P. Morgan Emerging Markets Bond Index Global (EMBI Global). J.P. Morgan Securities Inc. Emerging Markets Research, New York. https://faculty.darden.virginia.edu/liw/emf/embi.pdf

[9] https://www.imf.org/external/pubs/ft/wp/2015/wp15263.pdf

[10] https://www.invenomica.com.ar/riesgo-pais-embi-america-latina-serie-historica

[11] Cosset, J.C. and Roy, J. (1990) Forecasting Country Risk Ratings Using a Neural 
Network. Twenty-Third Annual Hawaii International Conference on System Sciences, Kailua Kona, 2-5 January 1990, 327-334.

[12] Rivero, C.R., Pucheta, J., Laboret, S. and Sauchelli, V. (2017) Energy Associated Tuning Method for Short-Term Series Forecasting by Complete and Incomplete Datasets. Journal of Artificial Intelligence and Soft Computing Research, 7, 5-16. https://doi.org/10.1515/jaiscr-2017-0001

[13] Hosking, J.R.M. (1984) Modeling Persistence in Hydrological Time Series Using Fractional Brownian Differencing. Water Resources Research, 20, 1898-1908. https://doi.org/10.1029/WR020i012p01898 https://agupubs.onlinelibrary.wiley.com/doi/epdf/10.1029/WR020i012p01898

[14] Marquardt, D. (1963) An Algorithm for Least-Squares Estimation of Nonlinear Parameters. SIAM Journal on Applied Mathematics, 11, 431-441.

https://doi.org/10.1137/0111030

[15] http://www.columbia.edu/ ad3217/fbm/hosking.c

[16] Mandelbrot, B.B. and Van Ness, J.W. (1968) Fractional Brownian Motions, Fractional Noises and Applications. SIAM Review, 10, 422-437.

https://doi.org/10.1137/1010093

[17] Dieker, T. (2004) Simulation of Fractional Brownian Motion. MSc Theses, University of Twente, Amsterdam.

[18] Abry, P., Flandrin, P., Taqqu, M.S. and Veitch, D. (2003) Self-Similarity and Long-Range Dependence through the Wavelet Lens. In: Doukhan, P., Oppenheim, G. and Taqqu, M., Eds., Theory and Applications of Long-Range Dependence, Birkhäuser, Basel, 527-556. 